\title{
Hospital admissions for acute anaphylaxis: time trend study
}

\author{
Aziz Sheikh, Bernadette Alves
}

Anecdotal evidence suggests that the incidence of acute anaphylaxis is increasing. ${ }^{1}$ Reasons for this supposed increase are poorly understood although a number of factors associated with the "Western lifestyle" have been implicated, such as changes in diet and the increasing use of therapeutic drugs. We investigated trends in hospital admissions for acute anaphylaxis using routinely collected national hospital discharge statistics from 1991-2 to 1994-5.

\section{Methods and results}

The hospital episode statistics database captures information on every admission to NHS hospitals in England. One primary diagnosis code and up to six secondary codes are recorded, the latter providing information on aetiology. We looked at hospital discharges occurring between 1 April 1991 and 31 March 1995 in which the primary ICD-9 (international classification of diseases, ninth revision) code was anaphylaxis (either anaphylactic shock (ICD-9 code 995.0) or anaphylactic shock due to serum (999.4)) together with a recorded aetiology. Data were analysed with the $\chi^{2}$ test for linear trends over time.

Over the four years there were 32.4 million discharge episodes. In 2424 of these the primary diagnosis was anaphylaxis. There was an increase from 5.6 instances of anaphylaxis per 100000 discharges in $1991-2$ to 10.2 in $1994-5$ ( $\left.\chi^{2}=141, \mathrm{P}<0.001\right)$ (figure).

Aetiology was recorded for 1248 of 2424 (51\%) discharges; the most common causes of anaphylaxis were therapeutic drugs $(772 / 1248 ; 62 \%)$, food (193/1248; 15\%), and insect venom (138/1248; 11\%). These percentages remained fairly constant throughout the four years. For some subcategories increases were apparent above the overall increase in anaphylaxis. These included the largest subcategory of food induced anaphylaxis (unspecified and other foods (E865.8, E865.9, $\mathrm{P}=0.03)$ and bacterial, biological, and other vaccines (E948, E949, $\mathrm{P}=0.003$ ).

\section{Comment}

This study identified an almost twofold increase between 1991 and 1995 in discharges from NHS hospitals in England with a primary diagnosis of anaphylaxis. Although many cases of anaphylaxis are likely to have been managed in accident and emergency departments without resulting in admission, studying discharges from hospitals is likely to provide useful information on the changing incidence, although it may be a substantial underestimate.

Possible confounding factors that may explain this rapid increase include changes in the quality of data from hospital episode statistics and a lowering of the threshold for admission. Hospital episode statistics have been used since 1987 but because it is widely recognised that the quality of data before 1990 was poor we decided to begin the study in $1991^{2}$; we confined our study to the period in which the ICD-9 was in use (use of ICD-10 began in 1995-6), reducing the

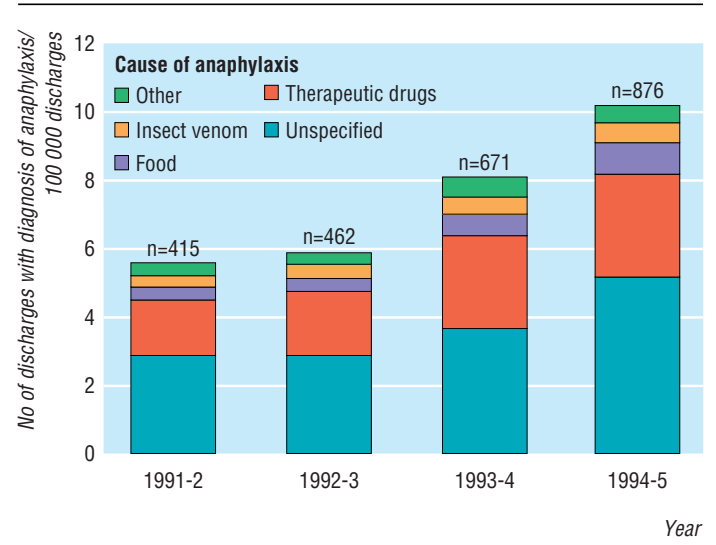

Number of hospital discharges with the primary diagnosis of anaphylaxis per 100000 episodes of hospital discharge and cause of anaphylaxis

possibility that systematic coding changes contributed to the observed increase. ${ }^{3}$ A lowering of the threshold for admission for anaphylaxis is an unlikely explanation for the findings since no national changes in anaphylaxis management were recommended during the study. Moreover, the increase in admissions for anaphylaxis was seen across all age groups, in both sexes, and within all four regions of England.

The proportion of anaphylaxis admissions associated with food allergy varied little; there was, however, evidence of a disproportionate increase in the subcategory of unspecified and other foods. This may reflect the increasing problem of peanut and nut allergy, ${ }^{45}$ although this cannot be concluded with confidence since the coding categories do not allow this food group to be studied individually. In view of the rapidly increasing incidence of anaphylaxis observed we recommend the development of mechanisms to increase the recording of substances triggering anaphylaxis together with more specific aetiological groupings of which nuts, latex, and individually named vaccines would be important examples.

We thank Dr Paul Aylin, Alex Bottle, Adrian Cook, Dr Sangeeta Dhami, and Professor Brian Hurwitz for their constructive comments on earlier drafts of this paper.

Contributors: AS conceived the study and is the guarantor. AS and BA designed the study, and BA analysed the data. The paper was written jointly by both authors.

Funding: AS is funded by a national primary care award from the NHS research and development programme.

Competing interests: None declared.

1 Ewan PW. Anaphylaxis. BMJ 1998;316:1442-5.

2 Hyndman SJ, Williams DR, Merill SL, Lipscombe JM, Palmer CR. Rates of admission to hospital for asthma. BMJ 1994;308:1596-1600.

3 NHS Centre for Coding and Classification. A guide to the use of tables of equivalence between ICD-9 and ICD-10. Loughborough: NHS Executive,
1995 .

4 Tariq SM, Stevens M, Matthews S, Ridout S, Twiselton R, Hide DW. Cohort study of peanut and tree nut sensitisation by age of 4 years. $B M J$ 1996;313:514-7.

5 Dibs SD, Baker MD. Anaphylaxis in children: a 5-year experience. Pediatrics 1997;99:e7. www.pediatrics.org/cgi/content/full/99/1/e7 (accessed December 1999).

(Accepted 15 March 2000)
Department of Primary Health Care and General Practice, Imperial College School of Medicine, London W2 1PG

Aziz Sheikh NHS R \& D national primary care training fellow Bernadette Alves health services researcher

Correspondence to: A Sheikh aziz.sheikh@ic.ac.uk BMJ 2000;320:1441 